\title{
WEAK AND STRONG CONVERGENCE FOR SOME OF NONEXPANSIVE MAPPINGS
}

\author{
Alireza Medghalchi and Shahram Saeidi*
}

\begin{abstract}
In this paper, we deal with a class of nonexpansive mappings with the property $D\left(\overline{c o} F_{\frac{1}{n}}(T), F(T)\right) \rightarrow 0$, as $n \rightarrow \infty$, where $D$ is the Hausdorff metric. We show that nonexpansive mappings with compact domains enjoy this property and give some examples of this kind of mappings with noncompact domains in $l^{\infty}$. Then we prove a nonlinear ergodic theorem, and a convergence theorem of mann's type for this kind of mappings.
\end{abstract}

\section{INTRODUCTION}

The first nonlinear ergodic theorem for nonexpansive mappings with bounded domains in a Hilbert space was established by Baillon [5]: Let $C$ be a nonempty closed convex subset of a Hilbert space $H$ and let $T$ be a nonexpansive mapping of $C$ into itself. If the set $F(T)$ of fixed points of $T$ is nonempty, then for each $x \in C$, the Cesaro means $S_{n}(x)=\frac{1}{n} \sum_{k=0}^{n} T^{k} x$ converge weakly to some $y \in F(T)$. Bruck [7] extended Baillon's theorem to a uniformly convex Banach space whose norm is Frechet differentiable. Before that, Edeleshtein [9] had obtained a nonlinear strong ergodic theorem for nonexpansive mappings with compact domains in a Banach space. Atsushiba and Takahashi [2] improved the Edelestein's theorem: Let $C$ be a nonempty compact convex subset of a strictly convex Banach space and let $T$ be a nonexpansive mapping of $C$ into itself. Then for each $x \in C$, the Cesaro means $S_{n}(x)=\frac{1}{n} \sum_{k=0}^{n} T^{k+h} x$ converge strongly to some $y \in F(T)$, uniformly in $h$.

The first purpose of this paper is to prove a nonlinear ergodic theorem for a specific class of nonexpansive mappings from a nonempty closed convex subset of a Bananch space into itself, which extends the Atsushiba and Takahashi's theorem. Our second goal is to prove a strong convergence theorem of mann's type [11] for this specific class of mappings.

Received October 26, 2006, accepted June 18, 2007.

Communicated by Mau-Hsiang Shih.

2000 Mathematics Subject Classification: 47H09, 47H10.

Key words and phrases: Fixed point, Nonexpansive mapping, Nonlinear ergodic theorem, Mapping of type $(\gamma)$, Strong convergence, Mann's type.

*Correspondng author. 


\section{Preliminaries}

Let $E$ be a real Banach space and let $C$ be a nonempty closed convex subset of $E$. A mapping $T: C \rightarrow C$ is said to be nonexpansive if $\|T x-T y\| \leq\|x-y\|$ for each $x, y \in C$. We denote by $F_{\varepsilon}(T)$ the $\varepsilon$-approximate fixed points of $T$; i.e. $F_{\varepsilon}(T)=\{x \in C:\|x-T x\| \leq \varepsilon\}$. If $C$ is bounded, then $F_{\varepsilon}(T) \neq \varnothing$ for each $\varepsilon>0$ (see [6]). A Banach space $E$ is said to be strictly convex if $\left\|\frac{x+y}{2}\right\|<1$ for $x, y \in E$ with $\|x\|=\|y\|=1$ and $x \neq y$. Let $E^{*}$ be the topological dual of $E$. The value of $x^{*} \in E^{*}$ at $x \in E$ will be denoted by $x^{*}(x)$. The open ball of radius $r$ centered at 0 is denoted by $B_{r}$. For a subset $A$ of $E$, we denote by $\overline{c o} A$ and $\bar{A}$ the closed convex hull and the closure of $A$, respectively. The distance from $x$ to $A$ is denoted by $\operatorname{dist}(x, A)$. We denote by $\Gamma$ the set of all strictly increasing, continuous convex functions $\gamma: \mathbb{R}^{+} \rightarrow \mathbb{R}^{+}$with $\gamma(0)=0$. For each $\gamma \in \Gamma$, a mapping $T: C \rightarrow C$ is said to be of type $(\gamma)$, if for every $x, y \in C$ and $\lambda \in[0,1]$, $\gamma(\|\lambda T x+(1-\lambda) T y-T(\lambda x+(1-\lambda) y)\|) \leq\|x-y\|-\|T x-T y\|$. Obviously, if $T$ is of type $(\gamma)$ for some $\gamma \in \Gamma$, then $T$ is nonexpansive and $F(T)$ is a convex set. Moreover if $C$ is also weakly compact, then $F(T) \neq \varnothing$ (see [10]). If $C$ is compact and $E$ is a strictly convex Banach space, then every nonexpansive mapping $T: C \rightarrow C$ is of type $(\gamma)$ (see $[2,7]$ ).

\section{Convergence to the Fixed Point Set}

First, we prove a lemma which we need in the following.

Lemma 3.1. Let $E$ be a locally convex space and $A_{1} \supseteq A_{2} \supseteq \cdots \supseteq A_{n} \supseteq$ $\cdots$ be a decreasing sequence of nonempty compact subsets. Then $\overline{c o}\left(\bigcap_{i=1}^{\infty} A_{i}\right)=$ $\bigcap_{i=1}^{\infty}\left(\overline{c o} A_{i}\right)$

Proof. Obviously $\overline{c o}\left(\bigcap_{i=1}^{\infty} A_{i}\right) \subseteq \bigcap_{i=1}^{\infty}\left(\overline{c o} A_{i}\right)$. Let $a \in \bigcap_{i=1}^{\infty}\left(\overline{c o} A_{i}\right)$ and $a \notin \overline{c o}\left(\bigcap_{i=1}^{\infty} A_{i}\right)$. Since $\bigcap_{i=1}^{\infty} A_{i} \neq \varnothing$, there exist $\varphi \in E^{*}, r \in \mathbb{R}$ and $\varepsilon>0$ such that

$$
\varphi(a)<r-\varepsilon \text { and } r+\varepsilon<\varphi(x)
$$

for every $x \in \overline{c o}\left(\bigcap_{i=1}^{\infty} A_{i}\right)$. Let $H_{r}=\{x \in E ; \varphi(x) \leq r\}$ and $A_{i}^{*}:=A_{i} \bigcap H_{r}$ for every $i \in \mathbb{N}$. By compactness of $A_{i}$ 's we conclude that $A_{i}^{*}$ 's are compact. We show that $A_{i}^{*} \neq \varnothing$ for every $i$. To see this, let $A_{j}^{*}=\varnothing$ for one $j \in \mathbb{N}$. Then $r<\varphi(b)$ for every $b \in A_{j}$ and so $r \leq \varphi(b)$ for every $b \in \overline{c o} A_{j}$. But $a \in \bigcap_{i=1}^{\infty}\left(\overline{c o} A_{i}\right)$, hence $a \in \overline{c o}\left(A_{j}\right)$ and we have $r \leq \varphi(a)$; but this is a contradiction to (1). Therefore 
$A_{1}^{*} \supseteq A_{2}^{*} \supseteq \cdots \supseteq A_{i}^{*} \supseteq \cdots$ is a decreasing sequence of nonempty compact subsets of $E$. Hence, $\left(\bigcap_{i} A_{i}\right) \bigcap H_{r}=\bigcap_{i}\left(A_{i} \bigcap H_{r}\right)=\bigcap_{i} A_{i}^{*} \neq \varnothing$. But, for $x \in\left(\bigcap_{i} A_{i}\right) \cap H_{r}$ we have $\varphi(x) \leq r$. This contradicts (1), and hence the assertion follows.

It should be noted that in general the convex hull of a compact set is not even closed (see [1, p. 173]. In a normed vector space, it is possible to apply Lemma 3.1 with the norm and weak topologies.

The following definition is well known:

Definition 3.2. Let $(M, \rho)$ be a complete metric space and $\Omega$ denotes the family of all nonempty, bounded closed subsets of $M$. For $X, Y \in \Omega$, set $d(X, Y)=$ $\sup \{\operatorname{dist}(y, X): y \in Y\}, d(Y, X)=\sup \{\operatorname{dist}(x, Y): x \in X\}$ and let $D(X, Y)=$ $\max \{d(X, Y), d(Y, X)\}$. Then $D$ provides a metric for $\Omega$ called the Hausdorff metric.

Let $C$ be a nonempty closed convex subset of a Banach space $E$ and $T$ : $C \rightarrow C$ be a nonexpansive mapping with $F(T) \neq \emptyset$. It is easy to verify that $D\left(\overline{c o} F_{\frac{1}{n}}(T), F(T)\right) \rightarrow 0$ as $n \rightarrow \infty$ iff $\operatorname{dist}\left(x_{n}, F(T)\right) \rightarrow 0$ as $n \rightarrow \infty$, for all sequences $\left\{x_{n}\right\}$ with $x_{n} \in \overline{c o} F_{\frac{1}{n}}(T), \forall n$. So, if $C$ is compact, it is easy to see that $D\left(\overline{c o} F_{\frac{1}{n}}(T), \bigcap_{i} \overline{c o} F_{\frac{1}{i}}(T)\right) \stackrel{n}{\rightarrow} 0$ as $n \rightarrow \infty$; and applying Lemma 3.1, we have $\bigcap_{i} \overline{c o} F_{\frac{1}{i}}(T)^{n}=\overline{c o} F(T)$. Therefore, we have shown

$$
D\left(\overline{c o} F_{\frac{1}{n}}(T), F(T)\right) \rightarrow 0
$$

as $n \rightarrow \infty$, in case that $F(T)$ is convex.

In this stage, we give some examples satisfying the convergence property above, however $C$ is not compact.

\section{Example 3.3.}

(i) Let $C=\prod_{i \in \mathbb{N}}[0,1] \subset l^{\infty}$. Then $C$ is not compact!. Now, let $T: C \rightarrow C$ be a nonexpansive mapping defined by $T\left(x_{1}, x_{2}, x_{3}, \ldots\right)=\left(f\left(x_{1}\right), 0,0, \ldots\right)$, where $f:[0,1] \rightarrow[0,1]$ is an arbitrary nonexpansive mapping. Since $\mathbb{R}$ is strictly convex and $[0,1]$ is compact, $f$ is of type $(\gamma)$ and $D\left(\overline{c o} F_{\frac{1}{n}}(f), F(f)\right) \rightarrow$ 0 . On the other hand, $F_{\frac{1}{n}}(T)=F_{\frac{1}{n}}(f) \times\left(\prod_{i \in \mathbb{N}-\{1\}}\left[0, \frac{1}{n}\right]\right)$. So $\overline{c o} F_{\frac{1}{n}}(T)=$ $\overline{c o} F_{\frac{1}{n}}(f) \times\left(\prod_{i \in \mathbb{N}-\{1\}}\left[0, \frac{1}{n}\right]\right)$ and $D\left(\overline{c o} F_{\frac{1}{n}}(T), F(T)\right) \rightarrow 0$, since $F(T)=$ $\left\{\left(x_{1}, 0,0, \ldots\right): x_{1} \in F(f)\right\}$. Also it is easy to see that $F(T)$ is compact and $T$ is of type $(\gamma)$.

(ii) Let $C=\prod_{i \in \mathbb{N}}\left[0, \frac{1}{2}\right] \subset l^{\infty}$ and $T\left(x_{1}, x_{2}, \ldots\right)=\left(\frac{x_{1}{ }^{2}}{2}, \frac{x_{2}{ }^{2}}{2}, \ldots\right)$. One notes that both $C$ and $T(C)$ are not compact!. Obviously $T$ is a nonexpansive 
mapping on $C$ and $F_{\frac{1}{n}}(T)=\prod_{i \in \mathbb{N}}\left[0,1-\sqrt{1-\frac{2}{n}}\right]$, for $n \geq 2$. Therefore, $D\left(\overline{c o} F_{\frac{1}{n}}(T), F(T)\right) \rightarrow 0$, where $F(T)=\{0\}$. The mapping $T$ is of type $(\gamma)$, where $\gamma$ is the identity mapping: Let $x, y \in C$ and $0 \leq \lambda \leq 1$. Then $\| \lambda T x+$ $(1-\lambda) T y-T(\lambda x+(1-\lambda) y) \|=\sup _{i}\left(\frac{1}{2} \mid \lambda x_{i}^{2}+(1-\lambda) y_{i}^{2}-\left(\lambda x_{i}+(1-\lambda)\right.\right.$ $\left.\left.y_{i}\right)^{2} \mid\right)=\frac{\lambda(1-\lambda)}{2} \sup _{i}\left(x_{i}-y_{i}\right)^{2}$. So, $\|T x-T y\|+\| \lambda T x+(1-\lambda) T y-T(\lambda x+$ $(1-\lambda) y) \|=\frac{1}{2} \sup _{i}\left|x_{i}^{2}-y_{i}^{2}\right|+\frac{\lambda(1-\lambda)}{2} \sup _{i}\left(x_{i}-y_{i}\right)^{2} \leq \frac{1}{2} \sup _{i}\left|x_{i}-y_{i}\right|+$ $\frac{1}{2} \sup _{i}\left|x_{i}-y_{i}\right|=\|x-y\|$, since $0 \leq x_{i}, y_{i} \leq \frac{1}{2}$ for each $i \in \mathbb{N}$. Therefore $T$ is of type $(\gamma)$, where $\gamma$ is the identity mapping. By an elementary computation we can show $T^{n}$ is also of type $(\gamma)$, for which $\gamma$ is the identity mapping.

(iii) Let $f:\left[0, \frac{1}{2}\right] \rightarrow\left[0, \frac{1}{2}\right]$ be an nonexpansive mapping of type $(\gamma)$, where $\gamma$ is the identity mapping, and $C$ be as in (ii). Define $T: C \rightarrow C$ by $T\left(x_{1}, x_{2}, \ldots\right)=$ $\left(f\left(x_{1}\right), \frac{x_{2}^{2}}{2}, \frac{x_{3}^{2}}{2}, \ldots\right)$. As in (ii), it is easy to show that for each $n, T^{n}$ is a nonexpansive mapping of type $(\gamma)$ such that $D\left(\overline{c o} F_{\frac{1}{n}}(T), F(T)\right) \rightarrow 0$, where $F(T)=\left\{\left(x_{1}, 0,0, \ldots\right): x_{1} \in F(f)\right\}$.

(iv) Let $C$ be as in (i) and $T: C \rightarrow C$ be a nonexpansive mapping defined by $T\left(x_{1}, x_{2}, x_{3}, \ldots\right)=\left(x_{1}, \frac{x_{2}^{2}}{2}, \frac{x_{3}^{2}}{2}, \ldots\right)$. Then we have $F(T)=\left\{\left(x_{1}, 0,0, \ldots\right)\right.$ : $\left.x_{1} \in[0,1]\right\}$ is a compact convex set. Also, we have $F_{\frac{1}{n}}(T)=[0,1] \times$ $\left(\prod_{i \in \mathbb{N}-\{1\}}\left[0,1-\sqrt{1-\frac{2}{n}}\right]\right)$, for $n \geq 2$. Hence, it is easy to note $D\left(\overline{c o} F_{\frac{1}{n}}(T)\right.$, $F(T)) \rightarrow 0$, as $n \rightarrow \infty$.

In the above examples $\overline{c o} F_{\frac{1}{n}}(T)$ 's are not compact; however, $F(T)$ 's are compact and we have $D\left(\overline{c o} F_{1}(T), F(T)\right) \rightarrow 0$, as $n \rightarrow \infty$. We can apply some results of this paper to examples like above.

\section{Cluster Point of Means}

The following lemmas are essential to our purpose.

Lemma 4.1. Let $C$ be a nonempty closed, convex subset of a Banach space $E$ and $T: C \rightarrow C$ be a nonexpansive mapping such that $F(T) \neq \varnothing$ and $D\left(\overline{c o} F_{1}(T), F(T)\right) \rightarrow 0$, as $n \rightarrow \infty$. Then, for any $\varepsilon>0$, there exists $\delta>0$ such that $\overline{c o} F_{\delta}(T) \subset F_{\varepsilon}(T)$.

Proof. Let $\varepsilon>0$. Since $D\left(\overline{c o} F_{\frac{1}{n}}(T), F(T)\right) \rightarrow 0$ there exists $\delta>0$ such that $\overline{c o} F_{\delta}(T) \subset F(T)+B_{\frac{\varepsilon}{2}}$. On the other hand, we have $F(T)+B_{\frac{\varepsilon}{2}} \subset F_{\varepsilon}(T)$. Hence the assertion follows. 
Lemma 4.2. Let $C, E$ and $T$ be as in Lemma 4.1. If $C$ is bounded and $T$ is of type $(\gamma)$, then for each $\eta>0$ there exists $\delta>0$ and $N>0$, such that for every sequence $\left\{x_{n}\right\}$ in $C$ satisfying $\left\|x_{n+1}-T x_{n}\right\| \leq \delta$ for all $n$,

$$
\frac{1}{n} \sum_{i=1}^{n} x_{i} \in F_{\eta}(T)
$$

for all $n \geq N$.

Proof. The proof is essentially the same as Theorem 1.3 of [8]. First, choose $\varepsilon>0$ using Lemma 4.1, so that $\overline{c o} F_{\varepsilon}(T) \subset F_{\frac{\eta}{3}}(T)$ and $\varepsilon d<\frac{\eta}{6}$ where $d=$ $\operatorname{diam} C$. We choose a natural number $p$ such that $d<p \frac{\varepsilon^{2}}{2}$. Next, put $q(t)=$ $\gamma^{-1}(2 t)+t$ and $q_{n}(t)=\gamma^{-1}\left(\frac{d}{n}+2 t\right)+t$ and choose $0<\delta<\frac{\eta}{3}$ so small that $q^{p-1}(\delta)<\frac{\varepsilon^{2}}{2}$. Finally, choose $N$ so large that $\frac{p}{N}<\varepsilon$ and $q_{n}^{p-1}(\delta)<\frac{\varepsilon^{2}}{2}$ for all $n \geq N$. Put $w_{i}=\frac{1}{p} \sum_{j=0}^{p-1} x_{j+i}$. Paralleling the proof of Lemma 1.5 of [7], we find $\frac{1}{n} \sum_{j=0}^{n-1}\left\|w_{j+1}-T w_{j}\right\| \leq q_{n}^{p-1}(\delta)$ provided $\left\|x_{i+1}-T x_{i}\right\| \leq \delta$ for all $i$. Obviously $\left\|w_{i+1}-w_{i}\right\| \leq \frac{d}{p}$ for all $i$. So by using the triangle inequality we have,

$$
\frac{1}{n} \sum_{i=0}^{n-1}\left\|w_{i}-T w_{i}\right\| \leq \varepsilon^{2}
$$

for every $n \geq N$. Put $A(n)=\left\{i \in \mathbb{Z}: 0 \leq i \leq n-1,\left\|w_{i}-T w_{i}\right\| \geq \varepsilon\right\}$ and $B(n)=\{0,1, \ldots, n-1\}-A(n)$. Then $\frac{|A(n)|}{n} \leq \varepsilon$ by (2). Also we have,

$$
\frac{1}{n} \sum_{i=0}^{n-1} x_{i}=\frac{1}{n} \sum_{i=0}^{n-1} w_{i}+\frac{1}{n p} \sum_{i=1}^{p-1}(p-i)\left[x_{i-1}-x_{n+i-1}\right]
$$

and $p \frac{d}{n} \leq p \frac{d}{N}<d \varepsilon$ for every $n \geq N$. Therefore,

$$
\left\|\frac{1}{n p} \sum_{i=1}^{p-1}(p-i)\left[x_{i}-x_{n+i-1}\right]\right\| \leq \frac{1}{n p} p^{2} d<d \varepsilon<\frac{\eta}{6}
$$

and so, $\frac{1}{n} \sum_{i=0}^{n-1} x_{i} \in\left[\frac{1}{n} \sum_{i=0}^{n-1} w_{i}\right]+B_{\frac{\eta}{6}}$. Fix $f \in F_{\varepsilon}(T)$. Then,

$$
\frac{1}{n} \sum_{i=0}^{n-1} w_{i}=\left[\frac{1}{n}|A(n)| f+\frac{1}{n} \sum_{i \in B(n)} w_{i}\right]+\left[\frac{1}{n} \sum_{i \in A(n)}\left(w_{i}-f\right)\right]
$$

and $\left\|\frac{1}{n} \sum_{i \in A(n)}\left(w_{i}-f\right)\right\| \leq \frac{|A(n)|}{n} d<\varepsilon d<\frac{\eta}{6}$. So, 


$$
\frac{1}{n} \sum_{i=0}^{n-1} x_{i} \in \operatorname{coF}_{\varepsilon}(T)+B_{\frac{\eta}{6}}+B_{\frac{\eta}{6}} \subset F_{\frac{\eta}{3}}(T)+B_{\frac{\eta}{3}} \subset F_{\eta}(T)
$$

for every $n \geq N$. This completes the proof.

Lemma 4.3. In Lemma 4.2 put $S_{n}=\frac{1}{n}\left(I+T+\cdots+T^{n-1}\right)$. Then $\lim _{n}\left\|S_{n}(y)-T S_{n}(y)\right\|=0$ uniformly in $y \in C$. Moreover, if $F(T)$ is compact (weakly compact), then for every sequence $\left\{y_{n}\right\}_{n \geq 1}$ in $C,\left\{S_{n}\left(y_{n}\right)\right\}_{n \geq 1}$ has a cluster point (weak cluster point) in $F(T)$.

Proof. Set $x_{n}=T^{n} y$ for each $n$ and $y \in C$, and apply Lemma 4.2 to conclude the first assertion. For the second assertion, let $F(T)$ be compact (weakly compact) and $\left\{y_{n}\right\}$ be an arbitrary sequence in $C$. We note $\operatorname{dist}\left(S_{n}\left(y_{n}\right), F(T)\right) \rightarrow 0$, as $n \rightarrow \infty$ by using the first part of this lemma. Then for each $k \geq 1$ there exist $n_{k}>k$ and $f_{k} \in F(T)$ with $\left\|S_{n_{k}}\left(y_{n_{k}}\right)-f_{k}\right\| \leq \frac{1}{k}$. Since $F(T)$ is compact (weakly compact), without lose of generality we can assume that $f_{k} \rightarrow f\left(f_{k} \rightarrow f\right)$ for some $f$, as $k \rightarrow \infty$. It is enough to conclude the result.

\section{ERGODIC THEOREMS}

By studying the proofs of Lemmas 2.2, 2.3 and 3.1 in [2], we obtain the following lemma:

Lemma 5.1. Let $C$ be a nonempty bounded closed convex subset of a Banach space $E, T: C \rightarrow C$ be a nonexpansive mapping such that $F(T) \neq \varnothing$ and $T^{n}$ is of type $(\gamma)$ for all $n$. Let $x \in C$. Then, there exists a sequence $\left\{i_{n}\right\}$ in $\mathbb{N}$ such that for each $z \in F(T)$,

$$
\lim _{n \rightarrow \infty}\left\|\frac{1}{n} \sum_{j=0}^{n-1} T^{j+i_{n}} x-z\right\| \text { exists. }
$$

Moreover if $\left\{i_{n}^{\prime}\right\}$ is a sequence in $\mathbb{N}$ such that $i_{n}^{\prime} \geq i_{n}$ for each $n \geq N$, then for every $z \in F(T)$,

$$
\lim _{n \rightarrow \infty}\left\|\frac{1}{n} \sum_{j=0}^{n-1} T^{j+i_{n}} x-z\right\|=\lim _{n \rightarrow \infty}\left\|\frac{1}{n} \sum_{j=0}^{n-1} T^{j+i_{n}^{\prime}} x-z\right\| .
$$

Recall that $E$ is said to satisfy Opial's condition, if for each sequence $\left\{x_{n}\right\}$ in $E$, the condition that the sequence $x_{n} \rightarrow x$ implies that

$$
\limsup _{n \rightarrow \infty}\left\|x_{n}-x\right\|<\limsup _{n \rightarrow \infty}\left\|x_{n}-y\right\|
$$

for all $y \in E$ with $y \neq x$. 
Theorem 5.2. Let $C$ be a nonempty closed convex subset of a Banach space $E$ and $T: C \rightarrow C$ be a nonexpansive mapping such that $F(T) \neq \varnothing$ and $T^{n}$ is of type $(\gamma)$ for all $n$ and $D\left(\overline{c o} F_{\frac{1}{n}}(T), F(T)\right) \rightarrow 0$, as $n \rightarrow \infty$. Let $x \in C$. Then,

(i) If $F(T)$ is compact, then $\frac{1}{n} \sum_{i=0}^{n-1} T^{i+h} x$ converges strongly to a fixed point of $T$ uniformly in $h \geq 0$.

(ii) If $F(T)$ is weakly compact and E satisfies Opial's condition, then $\frac{1}{n} \sum_{k=0}^{n}$ $T^{k+i_{n}} x$ converges weakly to some $y \in F(T)$, for a sequence $\left\{i_{n}\right\}$ like the sequence in Lemma 5.1.

Proof. Let $z$ be an arbitrary element of $F(T)$. Set $D=\{y \in C:\|y-z\| \leq$ $\|x-z\|\}$. We note that $x \in D, T(D) \subset D$ and $D$ is a bounded closed convex subset of $C$. So we can assume that $C$ is bounded. By Lemma 5.1, there exists a sequence $\left\{i_{n}\right\}$ in $\mathbb{N}$ such that for each $f \in F(T), \lim _{n \rightarrow \infty}\left\|\frac{1}{n} \sum_{j=0}^{n-1} T^{j+i_{n}} x-f\right\|$ exists. Now put $\left\{\Phi_{n}\right\}=\left\{\frac{1}{n} \sum_{j=0}^{n-1} T^{j+i_{n}} x\right\}$. We first prove (i). If $F(T)$ is compact, then $\left\{\Phi_{n}\right\}$ has a cluster point $y_{0}$ in $F(T)$ by Lemma 4.3.

Consequently, we have $\Phi_{n} \rightarrow y_{0}$; and from the last part of Lemma 5.1, $\frac{1}{n} \sum_{j=0}^{n-1} T^{j+h+i_{n}} x$ converges strongly to $y_{0}$ uniformly in $h \geq 0$. Let $\varepsilon>0$. Then, there exists $m \in \mathbb{N}$ such that $\left\|\frac{1}{n} \sum_{j=0}^{n-1} T^{j+h+i_{n}} x-y_{0}\right\|<\varepsilon$ for every $n \geq m$ and $h \in \mathbb{N} \cup\{0\}$. Then, it follows from the equality (3) that $\left\|\frac{1}{n} \sum_{i=0}^{n-1} T^{i+h} x-y_{0}\right\|$

$$
\begin{aligned}
& =\left\|\left[\frac{1}{n} \sum_{i=0}^{n-1} \frac{1}{m} \sum_{j=0}^{m-1} T^{i+j+h} x-y_{0}\right]+\left[\frac{1}{n m} \sum_{i=1}^{m-1}(m-i)\left(T^{i+h-1} x-T^{i+h+n-1} x\right)\right]\right\| \\
& \leq \frac{1}{n} \sum_{i=0}^{n-1}\left\|\frac{1}{m} \sum_{j=0}^{m-1} T^{j+h+i} x-y_{0}\right\|+\frac{1}{n m} \sum_{i=1}^{m-1}(m-i)\left\|T^{i+h-1} x-T^{i+h+n-1} x\right\| \\
& =\frac{1}{n} \sum_{i=0}^{i_{m}-1}\left\|\frac{1}{m} \sum_{j=0}^{m-1} T^{j+h+i} x-y_{0}\right\|+\frac{1}{n} \sum_{i=0}^{n-i_{m}-1}\left\|\frac{1}{m} \sum_{j=0}^{m-1} T^{j+h+i+i_{m}} x-y_{0}\right\| \\
& +\frac{1}{n m} \sum_{i=1}^{m-1}(m-i)\left\|T^{i+h-1} x-T^{i+h+n-1} x\right\| \leq \frac{i_{m} M}{n}+\frac{\left(n-i_{m}\right) \varepsilon}{n}+\frac{m M}{n},
\end{aligned}
$$

for every $n>i_{m}$ and $h \in \mathbb{N} \cup\{0\}$, where

$$
M=\sup \left\{\left\|T^{i} x-y_{0}\right\|: j \in \mathbb{N} \cup\{0\}\right\} .
$$

Since $\varepsilon>0$ is arbitrary, $\frac{1}{n} \sum_{i=0}^{n-1} T^{i+h} x$ converges strongly to $y_{0}$ uniformly in $h \in \mathbb{N} \cup\{0\}$, and so the proof of (i) is completed. To prove (ii) we assume $F(T)$ is weakly compact and $E$ satisfies Opial's condition. Then $\left\{\Phi_{n}\right\}$ has a weak cluster point $f$ in $F(T)$, by Lemma 4.3. We show $\Phi_{n} \rightarrow f$ as $n \rightarrow \infty$. Let $\Phi_{n_{k}} \rightarrow f_{1}$, $\Phi_{m_{k}} \rightarrow f_{2}$ and $f_{1} \neq f_{2}$. Since $f_{1}, f_{2} \in F(T)$, we put $r_{1}:=\lim _{n \rightarrow \infty}\left\|\Phi_{n}-f_{1}\right\|$ 
and $r_{2}:=\lim _{n \rightarrow \infty}\left\|\Phi_{n}-f_{2}\right\|$. By Opial's condition, we conclude

$$
\begin{aligned}
& r_{1}=\lim _{k \rightarrow \infty}\left\|\Phi_{n_{k}}-f_{1}\right\|<\lim _{k \rightarrow \infty}\left\|\Phi_{n_{k}}-f_{2}\right\|=r_{2} \\
& =\lim _{k \rightarrow \infty}\left\|\Phi_{m_{k}}-f_{2}\right\|<\lim _{k \rightarrow \infty}\left\|\Phi_{m_{k}}-f_{1}\right\|=r_{1},
\end{aligned}
$$

which is a contradiction. It means that $f_{1}=f_{2}$. This leads to the desired conclusion.

The following example shows that the condition $D\left(\overline{c o} F_{\frac{1}{n}}(T), F(T)\right) \rightarrow 0$ in Theorem 5.2 can not be omitted.

Example 5.3. Let $C$ and $E$ be as in Example 3.3 (i). Define $T: C \rightarrow C$ by $T\left(x_{1}, x_{2}, x_{3}, \ldots\right)=\left(\lambda_{1} x_{1}, \lambda_{2} x_{2}, \lambda_{3} x_{3}, \ldots\right)$, where $0 \leq \lambda_{i}<1$ for each $i \in \mathbb{N}$, and $\lim _{i \rightarrow \infty} \lambda_{i}=1$. Then $T$ is a nonexpansive mapping such that $T^{n}$ is of type $(\gamma)$ for all $n$ and $F(T)=\{0\}$ which is compact. Also, $F_{\frac{1}{n}}(T)=\prod_{i=1}^{\infty}\left(\left[0, \frac{1}{n\left(1-\lambda_{i}\right)}\right] \cap\right.$ $[0,1])$. So $\overline{c o} F_{\frac{1}{n}}(T) \stackrel{d}{\leftrightarrow} F(T)$. Now, by considering $x=(1,1, \ldots)$ in $C$ we have $\left\|\frac{1}{n} \sum_{i=1}^{n} T^{i} x\right\|=\sup _{k}\left(\frac{1}{n} \sum_{i=1}^{n} \lambda_{k}^{i}\right)=1$, since $\lim _{k \rightarrow \infty} \lambda_{k}=1$. So $\frac{1}{n} \sum_{i=1}^{n} T^{i} x$ does not converge to a member of $F(T)$.

\section{6. a Strong Convergence Theorem of Mann's Type}

In this section, using the iterative method of Mann's Type [11], we study how to find a fixed point of a nonexpansive mapping as in Theorem 5.2. Let $C$ be a nonempty closed convex subset of a Banach space $E$ and let $T$ be a nonexpansive mapping on $C$ with $F(T) \neq \varnothing$. Consider the following iteration scheme:

$$
x_{1}=x \in C \text { and } x_{n+1}=\alpha_{n} x_{n}+\left(1-\alpha_{n}\right) S_{n}\left(x_{n}\right)
$$

for every $n \in \mathbb{N}$, where $S_{n}=\frac{1}{n}\left(I+T+T^{2}+\cdots+T^{n-1}\right)$ and $\left\{\alpha_{n}\right\}$ is a sequence in $[0,1]$. For any $\omega \in F(T)$ we can prove

$$
\left\|x_{n+1}-\omega\right\| \leq\left\|x_{n}-\omega\right\|
$$

for every $n \in \mathbb{N}$ and hence $\lim _{n \rightarrow \infty}\left\|x_{n}-\omega\right\|$ exists (see [3]).

The following lemma is essential.

Lemma 6.1. Let $C$ be a nonempty closed convex subset of a Banach space $E$ and $T: C \rightarrow C$ be a nonexpansive mapping of type $(\gamma)$ such that $F(T) \neq \varnothing$ and $D\left(\overline{c o} F_{\frac{1}{n}}(T), F(T)\right) \rightarrow 0$, as $n \rightarrow \infty$. Let $\left\{\alpha_{n}\right\}$ be a sequence in $[0,1]$ such that $\sum_{n=1}^{\infty}\left(1-\alpha_{n}\right)=\infty$. Suppose that $x_{1}=x \in C$ and let $\left\{x_{n}\right\}$ be as in (4). Then

$$
\lim _{n \rightarrow \infty}\left\|T x_{n}-x_{n}\right\|=0 .
$$


Proof. As in Theorem 5.2 we can assume that $C$ is bounded. Fix $\varepsilon>0$ and set $M_{0}=\sup \{\|z\|: z \in C\}$. Then, by Lemma 4.1, there exists $\delta>0$ such that $\overline{c o} F_{\delta}(T) \subset F_{\varepsilon}(T)$. From Lemma 4.3, there exists $M \in \mathbb{N}$ such that $\left\|S_{n}(y)-T S_{n}(y)\right\|<\delta$ for every $n \geq M$ and $y \in C$. Thus

$$
S_{n}\left(x_{n}\right) \in F_{\delta}(T)
$$

for every $n \geq M$. We have for each $k \in \mathbb{N}$,

$$
x_{M+k}=\left(\prod_{i=M}^{M+k-1} \alpha_{i}\right) x_{M}+\left(1-\prod_{i=M}^{M+k-1} \alpha_{i}\right) y_{k}
$$

where

$$
\begin{gathered}
y_{k}=\frac{1}{1-\prod_{i=M}^{M+k-1} \alpha_{i}}\left(\sum_{j=M}^{M+k-2}\left(\left(\prod_{i=j+1}^{M+k-1} \alpha_{i}\right)\left(1-\alpha_{j}\right) S_{j}\left(x_{j}\right)\right)\right. \\
\left.+\left(1-\alpha_{M+k-1}\right) S_{M+k-1}\left(x_{M+k-1}\right)\right)
\end{gathered}
$$

(see $[3,4])$. Now, from

$$
\sum_{j=M}^{M+k-2}\left(\left(\prod_{i=j+1}^{M+k-1} \alpha_{i}\right)\left(1-\alpha_{j}\right)\right)+\left(1-\alpha_{M+k-1}\right)=1-\prod_{i=M}^{M+k-1} \alpha_{i}
$$

it follows that $y_{k} \in \operatorname{co}\left\{S_{n}\left(x_{n}\right): n \geq M\right\}$ and hence $y_{k} \in \operatorname{coF}_{\delta}(T) \subset F_{\varepsilon}(T)$ for each $k \in \mathbb{N}$, by (6). From the Abel-Dini theorem and $\sum_{i=M}^{\infty}\left(1-\alpha_{i}\right)=\infty$, there exists $p \in \mathbb{N}$ such that $\prod_{i=M}^{M+k-1} \alpha_{i}<\frac{\varepsilon}{2 M_{0}}$ for all $k \geq p$. From (7) we obtain

$$
\left\|x_{M+k}-y_{k}\right\|=\prod_{i=M}^{M+k-1} \alpha_{i}\left\|x_{M}-y_{k}\right\|<\frac{\varepsilon}{2 M_{0}} 2 M_{0}=\varepsilon
$$

for each $k \geq p$. Hence $\left\|T x_{M+k}-x_{M+k}\right\| \leq\left\|T x_{M+k}-T y_{k}\right\|+\left\|T y_{k}-y_{k}\right\|+$ $\left\|y_{k}-x_{M+k}\right\| \leq 2\left\|x_{M+k}-y_{k}\right\|+\left\|T y_{k}-y_{k}\right\| \leq 2 \varepsilon+\varepsilon=3 \varepsilon$ for every $k \geq p$. So $\lim _{n \rightarrow \infty}\left\|T x_{n}-x_{n}\right\|=0$.

Theorem 6.2. Let $C$ be a nonempty closed convex subset of a Banach space $E$ and $T: C \rightarrow C$ be a nonexpansive mapping of type $(\gamma)$ such that $F(T) \neq \varnothing$ and $D\left(\overline{c o} F_{1}(T), F(T)\right) \rightarrow 0$, as $n \rightarrow \infty$. Let $\left\{\alpha_{n}\right\}$ be a sequence in $[0,1]$ such that $\sum_{n=1}^{\infty}\left(1-\alpha_{n}\right)=\infty$. Suppose that $x_{1}=x \in C$ and $x_{n+1}=\alpha_{n} x_{n}+\left(1-\alpha_{n}\right) S_{n}\left(x_{n}\right)$ for every $n \in \mathbb{N}$. Then, $\left\{x_{n}\right\}$ converges strongly to a fixed point of $T$. 
Proof. By Lemma 6.1, $\lim _{n \rightarrow \infty}\left\|T x_{n}-x_{n}\right\|=0$. From the assumption $D\left(\overline{c o} F_{\frac{1}{n}}(T), F(T)\right) \rightarrow 0$, we have $\lim d\left(x_{n}, F(T)\right) \rightarrow 0$ as $n \rightarrow \infty$. Hereafter, we will prove that $\left\{x_{n}\right\}$ is a Cauchy sequence. For all $\epsilon>0$, there exists a natural number $N$ such that when $n \geq N d\left(x_{n}, F(T)\right)<\frac{\epsilon}{4}$. Specifically, $d\left(x_{N}, F(T)\right)<$ $\frac{\epsilon}{4}$. Thus there exists a point $y_{0}$ in $F(T)$ such that $\left\|x_{n}-y_{0}\right\| \leq\left\|x_{N}-y_{0}\right\|<\frac{\epsilon}{2}$ for each $n \geq N$, using (5) and the definition of $d\left(x_{N}, F(T)\right)$. It follows that for each $n \geq N$ and $m$ in $\mathbb{N},\left\|x_{n}-x_{n+m}\right\| \leq\left\|x_{n}-y_{0}\right\|+\left\|x_{n+m}-y_{0}\right\|<\epsilon$. This implies that $\left\{x_{n}\right\}$ is a Cauchy sequence. Because the space is complete, the sequence $\left\{x_{n}\right\}$ is convergent to a point that is a fixed point of $T$.

Remark 6.3. It is not assumed in Theorem 6.2 that $C$ be bounded nor $F(T)$ be compact.

\section{REFERENCES}

1. C. D. Aliprantis, K. C. Border, Infinite dimensional analysis, Springer-Verlage, Berlin-Heidelberg, 1999.

2. S. Atsushiba and W. Takahashi, A nonlinear strong ergodic theorem for nonexpansive mappings with compact domains, Math. Japonica., 52 (2000), 183-195.

3. S. Atsushiba and $\mathrm{W}$. Takahashi, $A$ weak convergence theorem for nonexpansive semigroups by the mann iteration process in Banach spaces, Proceeding of the International Conference on Nonlinear Analysis and Convex Analysis, (W. Takahashi and T. Tanaka, Eds.), World Scientific Publishers, pp. 102-109, 1999.

4. S. Atsushiba and W. Takahashi, Strong convergence theorems for one-parameter nonexpansive semi-groups with compact domains, in: Y. J. Cho, J. K. Kim and S. M. Kang (Eds.), Fixed Point Theory and Applications, Vol. 3, Nova Science Publisher, New York, 2002, pp. 15-31.

5. J. B. Baillon, Un theoreme de type ergodique pour les contractions non lineaires dans un espace de Hilbert, C. R. Acad. Sci. Paris Ser. A-B, 280 (1975), 1511-1514.

6. F. E. Browder, Nonlinear operators and nonlinear equations of evolution in Banach spaces, Proc. Symp. Pure Math., 18, part 2, 1976.

7. R. E. Bruck, A simple proof of the mean ergodic theorem for nonlinear contractions in Banach spaces, Israel J. Math., 32 (1979), 107-116.

8. R. E. Bruck, On the convex approximation property and the asymptotic behavior of nonlinear contractions in Banach spaces, Israel J. Math., 38 (1981), 304-314.

9. M. Edelestein, On non-expansive mappings of Banach spaces, Proc. Camb. Phill. Soc., 60 (1964), 439-447.

10. K. Goeble and W. A. Kirk, Topics in metric fixed point theory, Cambridge Univ. Press, 1990. 
11. W. R. Mann, Mean value methods in iteration, Proc. Amer. Math. Soc., 4 (1953), 506-510.

Alireza Medghalchi

Faculty of Mathematical Sciences and Computer Engineering,

Teacher Training University,

Tehran 15614,

Iran

E-mail: medghalchi@saba.tmu.ac.ir

Shahram Saeidi

Department of Mathematics,

University of Kurdistan,

Sanandaj, Kurdistan,

Iran

E-mail: sh.saeidi@uok.ac.ir shahram_saeidi@yahoo.com 